\title{
Structural magnetic resonance imaging and detailed motor assessment for chronic-phase patients after stroke
}

\author{
Ryo Ueda ${ }^{1,2}$, Naoki Yamada ${ }^{3,4}$, Masahiro Abo $^{3}$ and Atsushi Senoo ${ }^{1 *}$ \\ ${ }^{1}$ Department of Radiological Sciences, Graduate School of Health Sciences, Tokyo Metropolitan University, Tokyo 116-8551, Japan \\ ${ }^{2}$ Office of Radiation Technology, Keio University Hospital, Tokyo, Japan \\ ${ }^{3}$ Department of Rehabilitation Medicine, The Jikei University School of Medicine, Tokyo, Japan \\ ${ }^{4}$ Department of Rehabilitation, Shimizu Hospital, Tottori, Japan
}

\begin{abstract}
Background: Low-frequency repetitive transcranial magnetic stimulation of the nonlesional hemisphere combined with occupational therapy significantly improves motor function of the affected upper limb in post-stroke hemiparetic patients, but the recovery mechanism remains unclear.

Objective: We aimed to assess the relationship between the position of the stroke-induced lesion and subsequent motor function following a stroke rehabilitation program.

Methods: Patients with post-stroke upper extremity paralysis underwent combined low-frequency repetitive transcranial magnetic stimulation over the unaffected hemisphere and daily occupational therapy. The subject was more than 12 months after onset of stroke. Voxel-based lesion-symptom mapping (VLSM) and Atlasbased analysis was used.

Results: VLSM showed that the lesion on the thalamus was associated with improvement in motor function (corrected P $<0.05)$. Atlas-based analysis showed significant correlation between volume of damaged tissue in the corticospinal tract and degree of change in Fugl-Meyer Assessment scores ( $\mathrm{P}<0.05)$.

Conclusions: The results of the analysis indicated that the effect of our proposed treatment can be related to the position and size of the stroke-induced lesion. Especially, it seems that the stroke-induced lesion in the thalamic area connecting to the primary motor cortex plays an important role in such recovery in post-stroke hemiparetic patients.
\end{abstract}

\section{Introduction}

Prognosis is important for effective recovery of stroke patients. As functional recovery is affected by numerous biological and environmental factors [1]. such patients display highly variable degrees of recovery. A critical review of the literature indicated that several clinical and demographic variables may be valid predictors of general functional recovery, including neurologic factors such as consciousness at onset, disorientation in time and place, sitting balance, and severity of motor deficits [2].

Neurorehabilitation or rehabilitation based on brain science has recently gained popularity and involves novel procedures, such as repetitive transcranial magnetic stimulation (rTMS). rTMS is a noninvasive method used for stimulating selected brain areas to modulate cortical excitability and function. Local nerve activity is reduced at low-frequency rTMS $(\leq 1 \mathrm{~Hz})$, but it is enhanced at highfrequency rTMS $(\geq 10 \mathrm{~Hz})$ [3]. Low-frequency rTMS is currently being therapeutically used in chronic-phase stroke patients with paralysis of the upper limbs [4]. Since April 2009, we have implemented a 15day protocol in patients with post-stroke paralysis of the upper limbs that combines low-frequency rTMS on the unaffected hemisphere and occupational therapy (OT). This protocol is called the NovEl intervention Using Repetitive TMS and intensive Occupational Therapy (NEURO) and its use has resulted in significant improvements in motor function [5]. Neurophysiological evaluations aimed at examining the underlying mechanism of rTMS suggest an anti-spastic effect by reducing motor neuron excitability in the unaffected cerebral hemisphere [6].

Yamada, et al. used functional magnetic resonance imaging (fMRI) for elucidating the recovery mechanism of the NEURO intervention and found that it facilitates motor function recovery in the paralyzed upper limbs by inducing functional cortical reorganization of the brain [7]. However, individual patients recover their motor function to varying extents, and determining factors in the observed variation in recovery are still unclear. We hypothesized that this activation was related, at least in part, with reorganization of exercise-related brain structures. A necessary first step for assessing this hypothesis was identifying the factors, such as stroke location and severity, that were relevant to changes in the structure of the exercise-related regions prior to any treatment. The extent of motor recovery and functional outcome

${ }^{*}$ Correspondence to: Atsushi Senoo, Department of Radiological Sciences, Graduate School of Health Sciences, Tokyo Metropolitan University, 7-2-10 Higashi-Ogu, Arakawa-ku, Tokyo 116-8551, Japan, Tel: +81-3-3819-1211; Fax: +81-3-3819-1406; E-mail: senoo@tmu.ac.jp

Key words: voxel-based lesion-symptom mapping, motor function, stroke

Received: September 20, 2018; Accepted: September 26, 2018; Published: September 28, 2018 
after stroke varies among individuals. Several motor functions recover rapidly, while others remain as permanent deficits [8]. Clinical evidence suggests that the affected areas of the sensorimotor cortex influences the pattern of motor deficits [9]. Motor recovery and functional outcome after stroke may vary according to the location and size of brain lesions. Previous studies have evaluated the association of either only the size or only the location of brain lesions with motor and functional outcomes after stroke [10-12]. Some studies suggested that the location of lesions correlates with final outcomes $[10,11]$. While several studies suggested that the size of lesions correlates with final outcomes $[10,12]$. Therefore, the relationship between the location and size of brain lesions and motor and functional outcomes in stroke patients remains controversial. We hypothesized that factors determining the degree of motor function include the position and size of the stroke-induced lesion. To test this, we used voxel-based lesion-symptom mapping (VLSM) for predicting the clinical impact of these lesions at specific locations in the brain [13]. Using VLSM, differences in motor function scores obtained using tests, such as the Wolf Motor Function Test (WMFT), can be examined based on the presence/absence of damage in each voxel. VLSM has also been used for identifying important areas for recovery of motor function [14] and language comprehension [15] and recovery from spatial neglect in longitudinal studies [16]. In this study, we aimed to explain the motor deficit by lesion location and the response to our rTMS intervention.

\section{Methods}

\section{Subjects, admission, and discharge}

Fifty patients with post-stroke spastic upper limb hemiparesis were admitted to the Department of Rehabilitation Medicine, Shimizu Hospital, as inpatients. They were treated with 12 sessions of rTMS, daily OT, and daily self-training, except on holidays. Clinical characteristics of the patients are listed in Table 1 . Inclusion criteria were as follows: 1 ) at a probable plateau state with regard to the process of recovery from upper limb hemiparesis, as determined by serial OT introduced after the onset of stroke [no increase in the Fugl-Meyer Assessment [10] (FMA) score at the monthly evaluation during the latest 3 months]; 2) age at intervention is between 18 and 70 years; 3 ) suffered from stroke at least 12 months prior to study enrollment; 4) history of only one stroke; 5) no excessive spasticity in the affected upper limb ( $\leq 3$ on the modified Ashworth scale); 6) no cognitive impairment, as indicated by a pretreatment Mini Mental State Examination score of at least 26; 7) no intracranial metal clips or cardiac pacemakers; and 8) no history of convulsions for at least 1 year prior to study enrollment. Patients did not have symptoms of central pain or allodynia. We included only right-handed patients in this study considering the small number of left-handed patients. The study was approved by the Institutional

Table 1. Clinical characteristics of study patients $(n=50)$

\begin{tabular}{|l|l|l|}
\hline & $\begin{array}{l}\text { Dominant hand } \\
\text { paralysis (n= 25) }\end{array}$ & $\begin{array}{l}\text { Non-dominant hand } \\
\text { paralysis (n= 25) }\end{array}$ \\
\hline Age (years) & $60.6 \pm 7.2$ & $55.6 \pm 10.4$ \\
\hline $\begin{array}{l}\text { Gender, n (\%) } \\
\text { Males } \\
\text { Females }\end{array}$ & $\begin{array}{l}17(68) \\
8(32)\end{array}$ & $\begin{array}{l}20(80) \\
5(20)\end{array}$ \\
\hline $\begin{array}{l}\text { Type of stroke, n (\%) } \\
\text { Cerebral infarction } \\
\text { Intracerebral hemorrhage }\end{array}$ & $\begin{array}{l}7(28) \\
\text { Duration after stroke (years) }\end{array}$ & $6.1 \pm 4.2$ \\
\hline $\begin{array}{l}\text { Brunnstrom recovery stage, } \mathrm{n}(\%) \\
\text { Upper extremity } \\
\text { (III/IV/V/VI) }\end{array}$ & $\begin{array}{l}7(28) \\
18(72)\end{array}$ \\
\hline
\end{tabular}

Data are presented as number (percentage) of patients or mean \pm standard deviation.
Review Boards of Tokyo Metropolitan University and the Shimizu Hospital. Written informed consent was obtained from all the patients.

All participants had suffered from stroke $>1$ year prior to enrollment, and thus were long past the time frame for spontaneous recovery.

\section{Low-frequency rTMS and intensive OT}

Low-frequency rTMS was applied using a 70-mm Figure-8 coil attached to a MagPro R30 stimulator (MagVenture Company, Farum, Denmark). Focal 1-Hz rTMS was delivered at the site of the nonlesioned hemisphere that elicited the largest motor-evoked potentials in the first dorsal interosseous muscle of the unaffected upper limb. Each rTMS session comprised 2400 pulses for $40 \mathrm{~min}$. The intensity of stimulation was set at $90 \%$ of the resting motor threshold for the first dorsal interosseous muscle.

Intensive OT comprised $60 \mathrm{~min}$ of one-to-one training and $60 \mathrm{~min}$ of self-training, delivered by an occupational therapist after application of low-frequency rTMS. Each OT session began within $10 \mathrm{~min}$ of the application of rTMS. The one-to-one training program comprised shaping techniques, such as reaching forward to move a cup from one place to another; writing letters; drawing pictures using a pencil; and repetitive task practice techniques, such as clay squeezing and molding or pinching small coins. The self-training tasks were similar to the shaping techniques used in the one-to-one training program. We created a booklet with points of training and notes based on each patient's condition in the presence of an occupational therapist.

\section{Clinical evaluation of motor function}

We used FMA and WMFT for assessing assess upper limb motor function. These two assessments were performed by an occupational therapist on the day of admission and at discharge. Previous studies have investigated psychometric properties using these two scales and reported high inter-rater and test-retest reliabilities [17,18]. FMA for assessing upper limb motor function consists of 33 quantitative items. As each item is rated on a 3-point ordinal scale (0-2), the maximum motor performance score is 66 points. The 15 timed tasks of WMFT were applied and the sum of performance time of these tasks was calculated as the total time. When the task was not completed within $120 \mathrm{~s}$, the performance time of the task was recorded as $120 \mathrm{~s}$.

\section{Statistical analyses of motor score}

Motor function scores were analyzed for pre-intervention and postintervention data using the Shapiro-Wilk test, which showed a nonGaussian distribution. Therefore, the Wilcoxon signed-rank test was used for comparing changes in motor function after the intervention. All statistical analyses were performed using EZR software (Easy R, Saitama Medical Center, Jichi Medical University) [19].

\section{MRI}

All patients underwent three-dimensional T1-weighted imaging (3D-T1WI) on admission and following the 12-session intervention using a 1.5 T MRI system (ECHELON Vega, Hitachi medico, Tokyo, Japan). Parameters used for 3D-T1WI were rf-spoiled steady state gradient echo; repetition time, $7.7 \mathrm{~ms}$; echo time, $4.0 \mathrm{~ms}$; matrix, $256 \times$ 256 pixels; number of excitations, 1; field of view, $224 \mathrm{~mm}$; number of slices, 180 ; and voxel size, $0.875 \times 0.875 \times 1 \mathrm{~mm}$.

\section{Stroke injury site}

This analysis was performed after flipping the left and right side of the image such that all the lesion hemispheres were on the same side. 
Several previous studies have demonstrated that this analysis strategy increases statistical sensitivity $[12,20,21]$.

Structural images were preprocessed using Statistical Parametric Mapping software (SPM8: Wellcome Trust Center for Neuroimaging). The images were spatially normalized into the standard Montreal Neurological Institute (MNI) space using a unified segmentation algorithm optimized for use in patients with local brain lesions. Seghier, et al. demonstrated the utility of this procedure using real and artificial lesions [22]. Their analyses showed high sensitivity for detecting and delineating brain lesions with different sizes and textures and at different locations [22]. Their approach has important implications on the generation of lesion overlap maps for a given population and subsequent assessment of lesion-deficit mappings. The unified segmentation algorithm is a generative model that combines tissue segmentation, bias correction, and spatial normalization [22]. Although this algorithm was developed for use with brain images from normal subjects' brains, in 3D-T1WI images from patients, it outperforms the previous "gold standard," cost-function masking [23]. The lesion in the images from each patient was automatically identified using a modified unified segmentation algorithm and an outlier detection algorithm [24] set at default parameters. Accuracy of lesion delineation was visually verified in each slice to confirm the possibility and extent of automatic lesion identification and spatial normalization. Representative raw and lesion segmentation overlay images are depicted in Figure 1. These normalized lesions were subjected to statistical mapping analysis using VLSM algorithms implemented in the MRIcron NPM analyzing package (Columbia, SC, USA) [25] to determine brain areas where damage related to improvement in motor function had occurred. Preintervention WMFT scores and a change in these scores were used in the VLSM analysis. We used the nonparametric Brunner-Munzel test [26] to statistically compare voxel-based data, as present in NPM and MRIcron software [27]. Brunner-Munzel tests on continuous preintervention WMFT and FMA scores and changes in the WMFT score were performed on a voxel-by-voxel basis to compare performance in patients with a lesion compared with that in patients without a lesion. For appropriate Brunner-Munzel statistics, only voxels affected in at least 10 patients were tested [28]. To correct for multiple comparisons, we used a permutation-based family-wise error (FWE) correction with 1000 permutations with a correction threshold of $5 \%$. The results of Brunner-Munzel tests were color-coded and mapped on the MNI brain template by the software [27]. In this study, we also adopted an Atlas-based analysis. The anatomical localization of significantly affected regions, as identified by VLSM, was based on the Anatomical Automatic Labeling (AAL) Atlas and the Johns Hopkins University (JHU) Atlas. Briefly, the brain was parcellated based on anatomical labeling, including gray and white matter.

The overall distribution of lesions in our patients is described in Figure 2a. We show exactly which voxels were included in VLSM (Figure 2b). We scaled from 10 to 50 patients.

\section{Correlation between the motor function outcome and presence/absence of damage}

We evaluated correlation between the motor function outcome and presence/absence of damage for each voxel. In each voxel, correlation analysis was performed by replacing the presence/absence of damage in each individual with quantitative variables. To do this, we used the lesion mask of each individual on a standard brain image. The Spearman rank partial correlation coefficient for WMFT, degree of change in WMFT scores, and presence (or absence) of damage were calculated for each voxel. We categorized initial WMFT scores as covariates during partial correlation analysis. In addition, the Spearman rank partial correlation coefficient for FMA, degree of change in FMA scores, and presence (or absence) of damage were calculated for each voxel. We categorized initial FMA scores as covariates during partial correlation analysis. We mapped the correlation coefficient and $\mathrm{p}$ value of each voxel. Patients' statistical maps were masked inclusively (uncorrected $\mathrm{P}<0.01$ ). This statistical analysis was performed using MATLAB software (version R2014b; MathWorks, Natick, Massachusetts, USA).

\section{Magnitude of stroke damage}

We performed an additional region of interest analysis using atlases to investigate the correlation between region-wise damage and motor function. We also used atlases to investigate the correlation between region-wise damage and functional recovery. The magnitude of stroke damage after stroke was evaluated using an automated lesion identification algorithm [22]. For each participant, the magnitude of stroke damage was calculated in the Atlas mask of the significant region indicated by VLSM. The anatomical localization of the significantly affected regions identified by VLSM was based on the AAL Atlas and JHU Atlas. The size of the lesion mask within anatomical lesions of interest, such as the thalamic area connecting to the primary motor cortex and CST, were used for determining the lesion size. The Spearman rank partial correlation coefficient for lesion volume, according to the region-based Atlas, and improvement in motor function were also calculated. In addition, we analyzed the correlation between lesion volume (derived from the region-based Atlas) and pre-intervention WMFT scores and between lesion volume and preintervention FMA scores. These statistical analyses were performed using MATLAB software.

\section{Results}

\section{Clinical evaluation of motor function in the affected upper limb}

All patients completed the protocol without any adverse effects. The intervention resulted in significant improvements in motor function of the affected upper limb, as indicated FMA, WMFT. FMA scores increased from $45.3 \pm 8.9$ to $50.7 \pm 8.1$ points. In addition, WMFT performance time decreased from $496 \pm 349 \mathrm{~s}$ to $371 \pm 291 \mathrm{~s}$ (Wilcoxon signed-rank test, all $\mathrm{P}<0.001)$.

\section{Stroke injury site}

VLSM analyses revealed that pre-intervention WMFT scores were significantly associated with damage to the thalamus and corticospinal tract (FWE $\mathrm{P}<0.05$, Figure 2c). Further, pre-intervention FMA scores were also significantly associated with damage to the thalamus and corticospinal tract (FWE $\mathrm{P}<0.05$, Figure $2 \mathrm{~d}$ ). The degree of improvement in WMFT and FMA scores was also associated with lesions on a network centered in the thalamus areas and corticospinal tract (false discovery rate $\mathrm{P}<0.05$, Figure 2e, Figure $2 \mathrm{f}$ ).

\section{Oxford thalamic connectivity atlas and VLSM}

To examine the relationship between thalamic lesions and preintervention WMFT and FMA scores, we characterized cortical connectivity of the thalamic cluster using the Oxford Thalamic Connectivity Atlas [4], which provides probabilistic connectivity values based on diffusion tractography. Figure 3a shows VLSM results in red-yellow with connectivity probabilities for seven cortical regions. The strongest cortical connectivity is to the premotor cortex. 


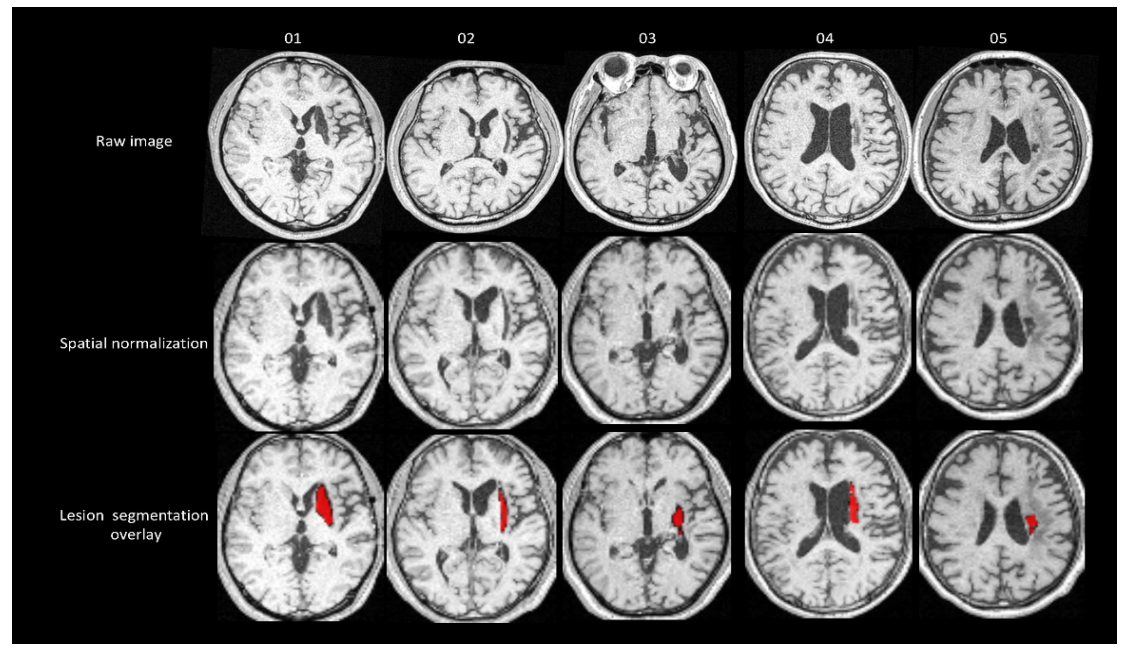

Figure 1. Raw image and lesion segmentation overlay

Representative images (raw image and lesion segmentation overlay) from study subjects. Accuracy of lesion delineation was visually verified for each slice to confirm plausibility and extent of automatic lesion identification and spatial normalization.

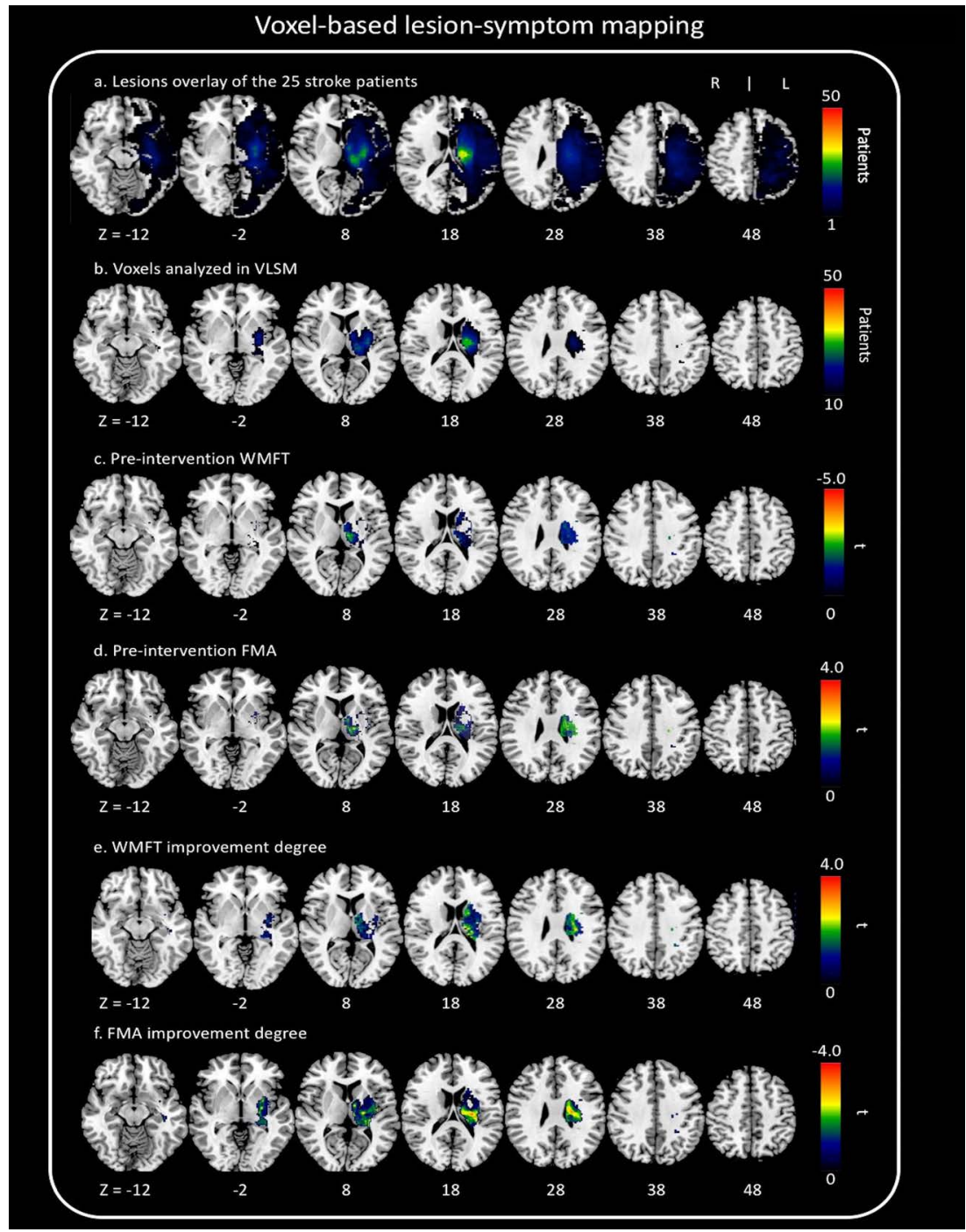

Figure 2. Voxel-based lesion-symptom mapping (VLSM)

(a) Overlap lesion plot from 50 stroke patients. The number of overlapping lesions is coded with colors ranging from dark blue $(\mathrm{n}=1)$ to light red $(\mathrm{n}=50$ patients). (b) Only voxels affected in at least 10 patients were included in the VLSM analysis. We showed exactly which voxels were included in the analysis. We scaled from 10 to 50 patients. (c) All voxels below $t=-3.8$ were significant at $\mathrm{P}=0.05$ (FWE correction). (d) All voxels above $\mathrm{t}=3.7$ were significant at $\mathrm{P}=0.05$ ( $\mathrm{FWE}$ correction). (e) All voxels above $\mathrm{t}=3.6$ were significant at $\mathrm{P}=0.05$ (false discovery rate correction). VLSM demonstrates that lesions on the thalamus are associated with motor function. (f) All voxels below $\mathrm{t}=-3.8$ were significant at $\mathrm{P}=0.05$ (false discovery rate correction). VLSM demonstrates that lesions on the thalamus are associated with motor function. 


\section{Correlation between the motor function outcome and presence/absence of damage}

The lesion in the pallidum, putamen, thalamus, superior parietal lobule, and inferior parietal lobule indicated a negative correlation with change in WMFT scores on partial correlation analysis $(\mathrm{P}<0.01$, Figure $3 \mathrm{~b}$ ). The lesion in the thalamus, insula, posterior limb of internal capsule, and middle frontal gyrus indicated a positive correlation with change in FMA scores on partial correlation analysis $(\mathrm{P}<0.01$, Figure $3 \mathrm{c})$.

\section{Magnitude of stroke damage}

On partial correlation analysis, the volume of the damaged tissue in the thalamic area connecting to the primary motor cortex did not significantly correlate with changes in WMFT scores $(\mathrm{r}=-0.09 ; \mathrm{P}=$ 0.27 ) (Figure 4a), but that correlated with changes in FMA scores $(r$ $=0.30 ; \mathrm{P}=0.02$ ) (Figure $4 \mathrm{c}$ ). The volume of damaged tissue in the corticospinal tract indicated a negative correlation trend with change in WMFT scores on partial correlation analysis $(\mathrm{r}=-0.20 ; \mathrm{P}=0.08)$ (Figure $4 \mathrm{~b})$, and that correlated with changes in FMA scores $(\mathrm{r}=0.25$; $\mathrm{P}=0.04)$ (Figure $4 \mathrm{~d}$ ).

The volume of damaged tissue in the thalamic area connecting to the primary motor cortex and pre-intervention WMFT scores also showed a significant positive correlation $(r=0.24 ; \mathrm{P}<0.05$, Figure $5 \mathrm{a})$. The volume of damaged tissue in the thalamic area connecting to the primary motor cortex and pre-intervention FMA scores also showed a significant negative correlation $(r=-0.29 ; \mathrm{P}<0.05$, Figure $5 \mathrm{c})$. The volume of damaged tissue in the corticospinal tract and pre-intervention WMFT scores were not significantly correlated $(r=0.0039 ; \mathrm{P}=0.49$, Figure $5 b$ ). The volume of damaged tissue in the corticospinal tract and pre-intervention FMA scores were not significantly correlated $(\mathrm{r}=$ $-0.079 ; \mathrm{P}=0.29$, Figure $5 \mathrm{~d}$ ).

\section{Discussion}

Low-frequency rTMS improves motor function in the paralyzed upper limbs of stroke patients by suppressing the activity of the unaffected hemisphere, which results in reduced interhemispheric inhibition and greater brain activity in the affected hemisphere [29]. Furthermore, studies using fMRI and TMS mapping have reported that functional cortical reorganization in the affected hemisphere occurs through intensive rehabilitation [30,31]. Based on these studies, we designed and implemented a protocol that combines low-frequency rTMS and intensive OT to promote functional cortical reorganization of the affected hemisphere. Results of the present study demonstrate significant improvement in motor function following this novel treatment strategy.

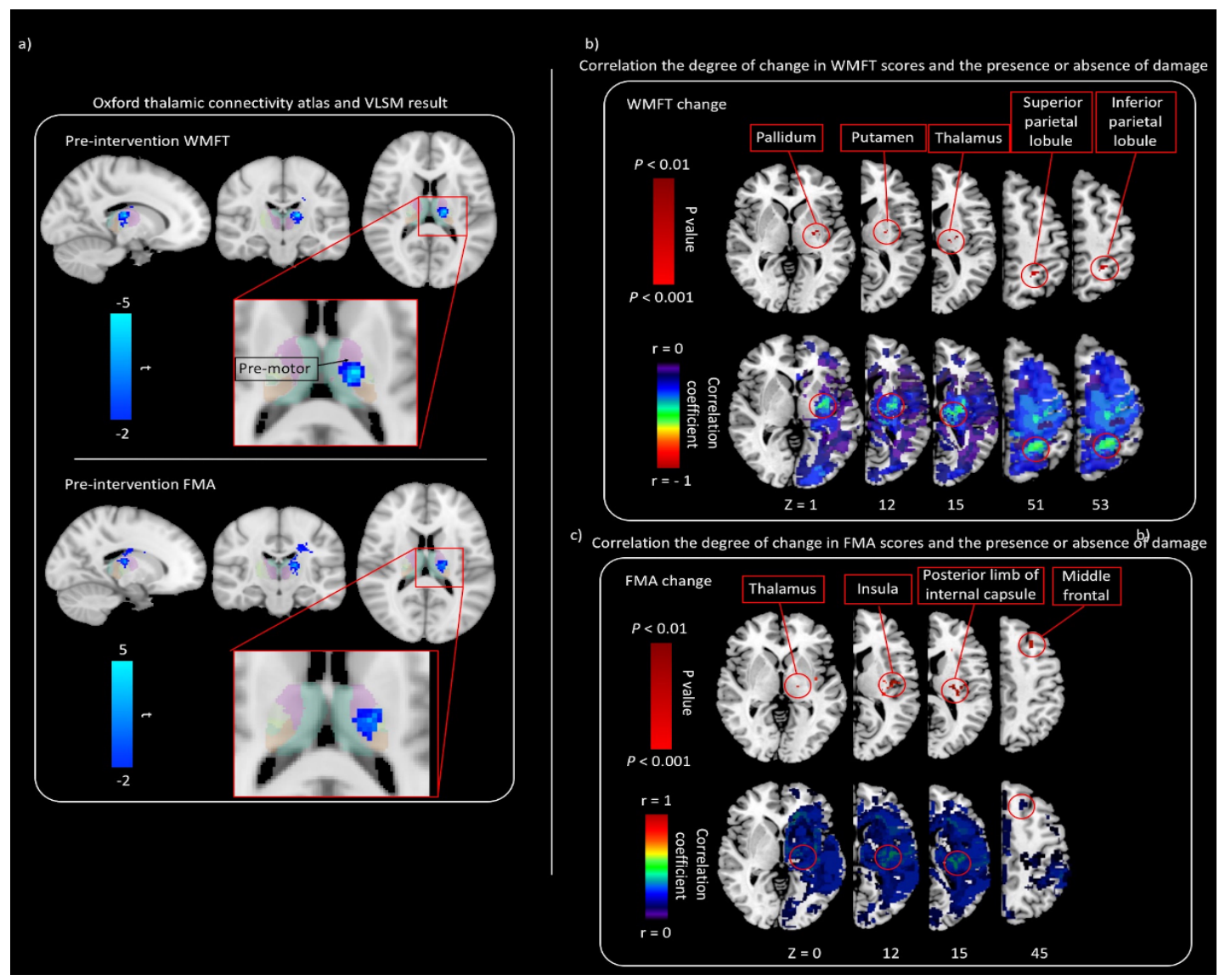

Figure 3. Relationship between the motor function and presence/absence of damage

(a) Regions with a strong relationship between pre-intervention WMFT scores and the lesion are shown. The thalamic cluster (colored in red-yellow) is displayed along with probable regions of cortical connectivity, based on the Oxford Thalamic Connectivity Atlas. The predominant relationship is to the premotor cortex. (b) Correlation between the degree of change in WMFT scores and presence/absence of damage. The correlation map shows negative correlation between the degree of change in WMFT scores and lesion. Pixels with significant correlation are located in the pallidum, putamen, thalamus, superior parietal lobule, and inferior parietal lobule. (c) Correlation between the degree of change in FMA scores and presence/absence of damage. The correlation map shows positive correlation between the degree of change in FMA scores and lesion. Pixels with significant correlation are located in $t$ the thalamus, insula, posterior limb of internal capsule, and middle frontal gyrus. 
a)

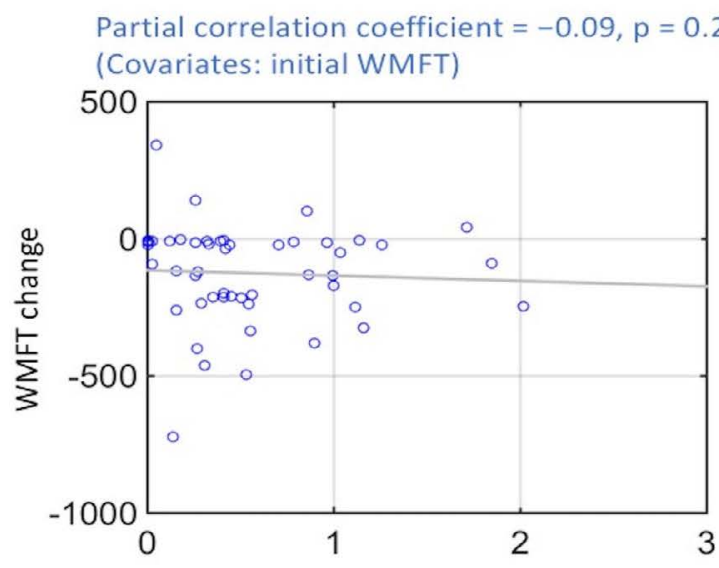

Damaged volume of the thalamic area connecting to the primary motor cortex $(\mathrm{ml})$

c)

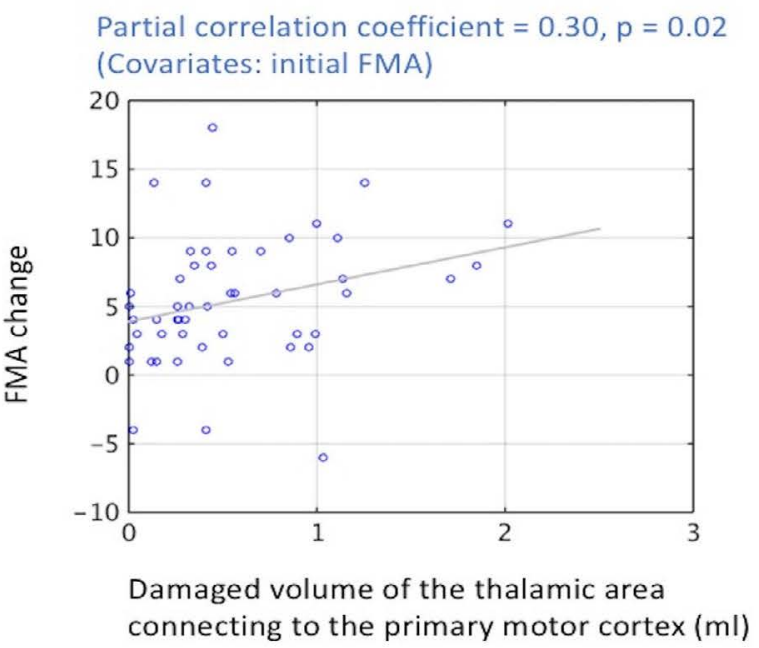

b)

Partial correlation coefficient $=-0.20, p=0.08$ (Covariates: initial WMFT)

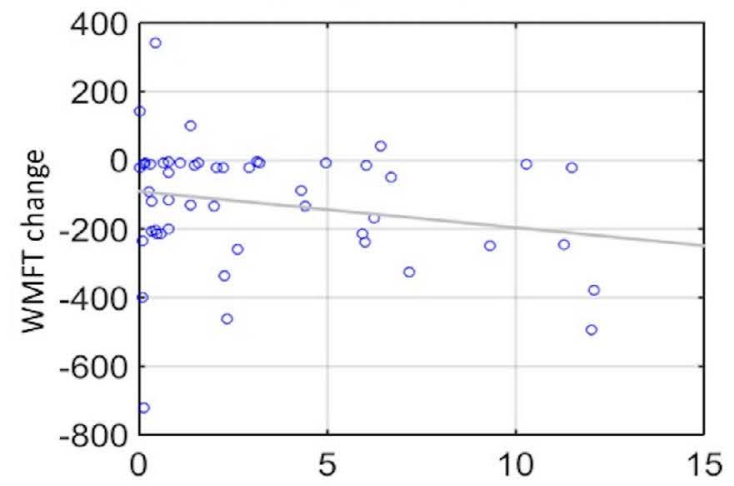

Damaged volume of the corticospinal tract $(\mathrm{ml})$

d)

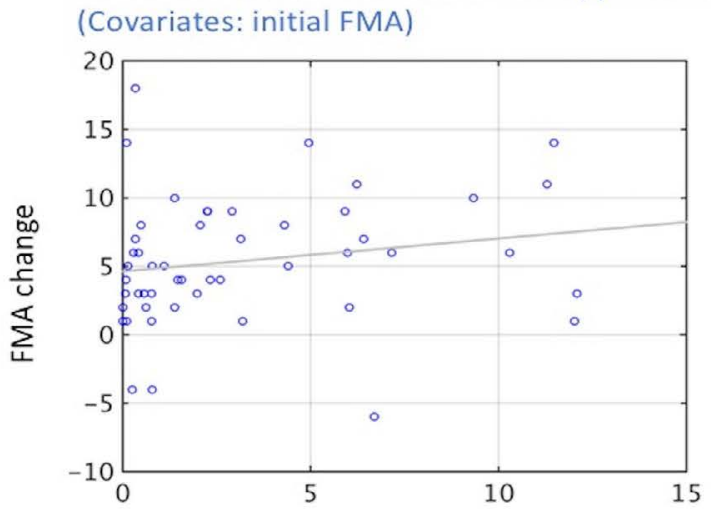

Damaged volume of the corticospinal tract $(\mathrm{ml})$

Figure 4. Correlations between improvement in motor function and lesion size

(a) Significant negative correlation was not observed between volume of damaged thalamic area connecting to the primary motor cortex and degree of change in WMFT scores ( $<0.05$ ) (b) A negative correlation trend was observed between the volume of tissue damage to the corticospinal tract and degree of change in WMFT scores ( $\mathrm{P}=0.08$ ). (c) Significant positive correlation was observed between volume of damaged thalamic area connecting to the primary motor cortex and degree of change in FMA scores $(\mathrm{P}<0.05$ ). (d) A positive correlation was observed between the volume of tissue damage to the corticospinal tract and degree of change in FMA scores $(\mathrm{P}<0.04)$.

To elucidate the relationship between anatomical location of the stroke-induced lesion and motor function, we performed VLSM analysis of pre-intervention WMFT and FMA scores. WMFT and FMA scores were significantly correlated with damage to the area encompassing the thalamus and corticospinal tract. Furthermore, we also investigated which lesion sites were associated with a positive rehabilitation outcome, aiming to identify brain regions that are related to good recovery outcomes. Therefore, we mapped recovery scores (WMFT post - WMFT pre and FMA post - FMA pre) via VLSM. Our results show that brain damage was associated with changes in WMFT and FMA scores. Post-stroke motor disturbance depends on the position of the lesion to a greater extent than the size [32]. Similarly, we showed that thalamic lesions affected motor function in the upper extremities [33]. Furthermore, damage to the thalamus, including damage to the posterior limb of the internal capsule, has been shown to have a negative impact on motor function recovery [34]. There is significant previous study examining the relationship between lesion location and post-stroke motor recovery or other general predictors of recovery [35-38]. The previous anatomical correlates of motor outcomes after stroke have concentrated on quantifying damage to the corticospinal tract $[33,35]$. The results of the current study are consistent with those of these previous reports showing that damage to the corticospinal tract correlates well with negative outcomes in motor function.

Next, we analyzed the correlation between the extent of damage to the thalamic tissue (volume) and pre-intervention WMFT and FMA scores. We found that a higher volume of damage to the thalamus was associated with higher pre-intervention WMFT scores and smaller 
a)

Correlation coefficient $=0.24, p=0.049$

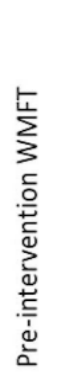

c)

Correlation coefficient $=-0.29, \mathrm{p}=0.021$

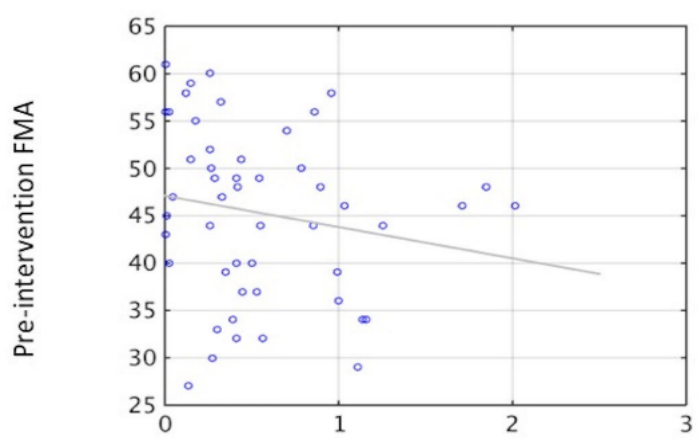

Damaged volume of the thalamic area connecting to the primary motor cortex $(\mathrm{ml})$

Damaged volume of the thalamic area connecting to the primary motor cortex $(\mathrm{ml})$ b)

\section{Correlation coefficient $=0.0039, p=0.49$}

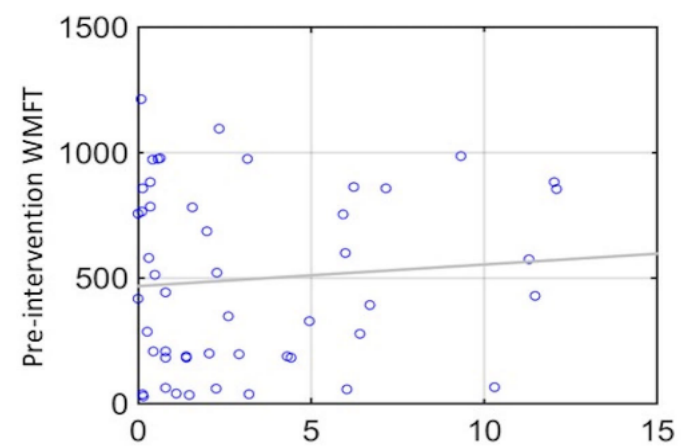

Damaged volume of the corticospinal tract (ml)

Figure 5. Correlation between pre-intervention WMFT and lesion size and between pre-intervention FMA and lesion size

Significant positive correlations are observed between volume of damaged thalamic area connecting to the primary motor cortex and pre-intervention WMFT scores $(\mathrm{P}<0.05)$. (b) Volume of damaged corticospinal tract tissue and pre-intervention WMFT scores were not correlated $(\mathrm{P}=0.49)$. (c) Significant negative correlations are observed between volume of damaged thalamic area connecting to the primary motor cortex and pre-intervention FMA scores $(\mathrm{P}<0.05)$. (d) Volume of damaged corticospinal tract tissue and pre-intervention FMA scores were not correlated $(\mathrm{P}=0.29)$

pre-intervention FMA scores. Specifically, pre-intervention WMFT and FMA scores demonstrated a greater relationship with the area of the thalamus connected to premotor areas. This particular thalamic area connects to the primary motor cortex on the affected side and is responsible for motion control and strongly influences paralysis. Therefore, our results suggest that a patient with greater damage to the thalamic area connected to premotor areas has reduced motor function in the upper limb, following a chronic-phase stroke. The most prolonged and unremitting paralysis occurs following hemispheric lesions [36] that destroy not just corticospinal fibers but also fiber tracts linking motor cortices with movement-related neuronal populations in the thalamus and striatum. Our current findings are consistent with those of this previous research. Reorganization of the cortex post stroke is dependent not only on the lesion site but also on remote brain areas that have structural connections with the area damaged [37-39]. Premotor cortex may be adversely affected if thalamic regions projecting to premotor cortex have an influence.
As VLSM analyzes the relationship between lesion location and symptoms, we hypothesized that it is possible to objectively reveal the relationship between the size of the stroke-induced lesion and degree of motor function by performing Atlas-based analysis. An Atlas-based approach ameliorates these shortcomings by grouping anatomically related voxels within the same anatomical unit. This systematically reduces the location information from hundreds of thousands of voxels to a limited number of regions of interest. Therefore, we analyzed the correlation between improvement in motor function (WMFT and FMA) and lesion size of the tissue in the thalamic area connecting to the primary motor cortex and corticospinal tract. The results of this study showed that improvement in motor function tended to be better in patients with a larger lesion volume in the corticospinal tract than in those with a smaller lesion volume in the corticospinal tract. Furthermore, in this correlation analysis, the state of motor function prior to intervention was included as a covariate. Our results also showed that a combination of rTMS and OT may effectively 
improve motor function in patients with motor paralysis due to largevolume lesions in the corticospinal tract. Thus, it is possible that the degree of improvement in motor function could be predicted by accurately evaluating the anatomical location of a stroke-induced lesion. Correlation of the presence/absence of damage for each voxel between the motor function outcome could be useful in determining the responsible spatial locations. As a result, change in the WMFT or FMA score was correlated with damage to the area encompassing the pallidum, putamen, thalamus, superior parietal lobule, inferior parietal lobule, insular, and posterior limb of internal capsule. These are areas involved in some aspects of motor function or sensory, such as planning movements and/or integrating visual information with movement to facilitate actions such as reaching and grasping. Therefore, we can predict the degree of improvement in motor function by investigating these anatomical areas. However, without a control group, we can only speculate on the potential mechanism of action of combined TMS/OT treatment based on present data.

Our study has several limitations. First, this study did not have a control group, which would be essential for confirming beneficial effects of our protocol. Therefore, a randomized controlled study that includes a control group is needed to confirm our findings. Second, we used an automated lesion detection method for identifying lesions. This introduces potential error as patients with large volumes of damaged tissue were excluded from the present study. Furthermore, the study sample was limited to patients with moderate paralysis, and thus, patients with severe paralysis were excluded. Other limitations include a low proportion of females and patients with cerebral infarction in the sample and the lack of blinding. Thus, our findings should be confirmed in a randomized controlled trial with a larger patient cohort in whom changes in motor function, including every day quality of life, are evaluated outside the treatment setting [40-42].

\section{Conclusions}

We examined the relationship between the motor function and position and size of the stroke-induced lesion, as identified by MRI, in patients with upper limb paralysis following chronic stroke. We showed that lesions in the thalamic tissue connected to the premotor areas are associated with upper limb motor function. These results can provide important guidelines for managing clinical symptoms of patients suffering from chronic-phase stroke.

\section{Disclosure Statement}

Authors declare that they have no conflicts of interest.

\section{Acknowledgments}

We thank Editage for English language editing.

\section{References}

1. Bach-y-Rita P, Bach-y-Rita EW (1990) Biological and psychosocial factors in recovery from brain damage in humans. Can J Psychol 44: 148-165. [Crossref]

2. Kwakkel G, Wagenaar RC, Kollen BJ, Lankhorst GJ (1996) Predicting disability in stroke--a critical review of the literature. Age Ageing 25: 479-489. [Crossref]

3. Maeda F, Keenan JP, Tormos JM, Topka H, Pascual-Leone A (2000). Interindividual variability of the modulatory effects of repetitive transcranial magnetic stimulation on cortical excitability. Exp Brain Res 133: 425-430.

4. Kakuda W, Abo M, Kaito N, Ishikawa A, Taguchi K, et al. (2010) Six-day course of repetitive transcranial magnetic stimulations plus occupational therapy for post-stroke patients with upper limb hemiparesis: a case series study. Disabil Rehabil 32: 801-807.

5. Kakuda W, Abo M, Shimizu M, Sasanuma J, Okamoto T, et al. (2012) A multi-center study on low-frequency rTMS combined with intensive occupational therapy for upper limb hemiparesis in post-stroke patients. J Neuroeng Rehabil 9: 4. [Crossref]
6. Kondo T, Kakuda W, Yamada N, Shimizu M, Hagino H, et al. (2013) Effect of low-frequency rTMS on motor neuron excitability after stroke. Acta Neurologica Scandinavica 127: 26-30.

7. Yamada N, Kakuda W, Senoo A, Kondo T, Mitani S, et al. (2013) Functional cortical reorganization after low-frequency repetitive transcranial magnetic stimulation plus intensive occupational therapy for upper limb hemiparesis: evaluation by functional magnetic resonance imaging in poststroke patients. Int J Stroke 8: 422-429.

8. Warabi T, Inoue K, Noda H, Murakami S (1990) Recovery of voluntary movement in hemiplegic patients. Correlation with degenerative shrinkage of the cerebral peduncles in CT images. Brain 113: 177-189. [Crossref]

9. Kunesch E, Binkofski F, Steinmetz H, Freund HJ (1995) The pattern of motor deficits in relation to the site of stroke lesions. Eur Neurol 35: 20-26. [Crossref]

10. Beloosesky Y, Streifler JY, Burstin A, Grinblat J (1995) The importance of brain infarct size and location in predicting outcome after stroke. Age Ageing 24: 515-518. [Crossref]

11. Chaudhuri G, Harvey RF, Sulton LD, Lambert RW (1988) Computerized tomography head scans as predictors of functional outcome of stroke patients. Archives of physical medicine and rehabilitation 69: 496-498.

12. van Everdingen KJ, van der Grond J, Kappelle LJ, Ramos LM, Mali WP (1998) Diffusion-weighted magnetic resonance imaging in acute stroke. Stroke 29: 1783-1790. [Crossref]

13. Bates E, Wilson SM, Saygin AP, Dick F, Sereno MI, et al. (2003) Voxel-based lesionsymptom mapping. Nature neuroscience 6: 448-450.

14. Lo R, Gitelman D, Levy R, Hulvershorn J, Parrish T (2010) Identification of critical areas for motor function recovery in chronic stroke subjects using voxel-based lesion symptom mapping. Neuroimage 49: 9-18.

15. Magnusdottir S, Fillmore P, den Ouden DB, Hjaltason H, Rorden C, et al. (2013) Damage to left anterior temporal cortex predicts impairment of complex syntactic processing: a lesion-symptom mapping study. Hum Brain Mapp 34: 2715-2723.

16. Karnath HO, Rennig J, Johannsen L, Rorden C (2011) The anatomy underlying acute versus chronic spatial neglect: a longitudinal study. Brain 134: 903-912.

17. Platz T, Pinkowski C, van Wijck F, Kim IH, di Bella P, et al. (2005) Reliability and validity of arm function assessment with standardized guidelines for the Fugl-Meyer Test, action research arm test and box and block test: a multicentre study. Clin Rehabil 19: 404-411.

18. Wolf SL, Thompson PA, Morris DM, Rose DK, Winstein CJ, et al. (2005) The EXCITE trial: attributes of the Wolf Motor Function Test in patients with subacute stroke. Neurorehabil Neural Repair 19: 194-205.

19. Kanda Y (2013) Investigation of the freely available easy-to-use software 'EZR' for medical statistics. Bone Marrow Transplant 48: 452-458. [Crossref]

20. Meyer S, Kessner SS, Cheng B, Bönstrup M, Schulz R, et al. (2016) Voxel-based lesion-symptom mapping of stroke lesions underlying somatosensory deficits. Neuroimage Clin 10: 257-266.

21. Takenobu Y, Hayashi T, Moriwaki H, Nagatsuka K, Naritomi H, et al. (2016) Motor recovery and microstructural change in rubro-spinal tract in subcortical stroke. Neuroimage Clin 4: 201-208.

22. Ashburner J, Friston KJ (2005) Unified segmentation. Neuroimage 26: 839-851. [Crossref]

23. Crinion J, Ashburner J, Leff A, Brett M, Price C, Friston K (2007) Spatial normalization of lesioned brains: performance evaluation and impact on fMRI analyses. Neuroimage 37: 866-875.

24. Seghier ML, Ramlackhansingh A, Crinion J, Leff AP, Price CJ (2008) Lesion identification using unified segmentation-normalisation models and fuzzy clustering. Neuroimage 41: 1253-1266.

25. Rorden C, Karnath HO, Bonilha L (2007) Improving lesion-symptom mapping. J Cogn Neurosci 19: 1081-1088. [Crossref]

26. Brunner E, Munzel U (2000) The nonparametric Behrens-Fisher problem: asymptotic theory and a small-sample approximation. Biometrical Journal: Journal of Mathematical Methods in Biosciences 42: 17-25.

27. Rorden C, Brett M (2000) Stereotaxic display of brain lesions. Behav Neurol 12: 191200. [Crossref]

28. Medina J, Kimberg DY, Chatterjee A, Coslett HB (2010) Inappropriate usage of the Brunner-Munzel test in recent voxel-based lesion-symptom mapping studies. Neuropsychologia 48: 341-343. 
Ueda R (2018) Structural magnetic resonance imaging and detailed motor assessment for chronic-phase patients after stroke

29. Segonne F, Dale AM, Busa E, Glessner M, Salat D, et al. (2004) A hybrid approach to the skull stripping problem in MRI. Neuroimage 22: 1060-1075.

30. Hummel FC, Cohen LG (2006) Non-invasive brain stimulation: a new strategy to improve neurorehabilitation after stroke. The Lancet Neurology 5: 708-712.

31. Liepert J, Bauder H, Wolfgang HR, Miltner WH, Taub E, Weiller C (2000) Treatment induced cortical reorganization after stroke in humans. Stroke 31: 1210-1216.

32. Levy CE, Nichols DS, Schmalbrock PM, Keller P, Chakeres DW (2001) Functional MRI evidence of cortical reorganization in upper-limb stroke hemiplegia treated with constraint-induced movement therapy. Am J Phys Med Rehabil 80: 4-12.

33. Zhu LL, Lindenberg R, Alexander MP, Schlaug G (2010) Lesion load of the corticospinal tract predicts motor impairment in chronic stroke. Stroke 41: 910-915. [Crossref]

34. Verret JM, Lapresle J (1986) [Motor symptomatology of the thalamus]. Rev Neurol (Paris) 142: 368-374. [Crossref]

35. Rondina JM, Filippone M, Girolami M, Ward NS (2016) Decoding post-stroke motor function from structural brain imaging. Neuroimage Clin 12: 372-380.
36. Shelton FN, Reding MJ (2001) Effect of lesion location on upper limb motor recovery after stroke. Stroke 32: 107-112. [Crossref]

37. Zarahn E, Alon L, Ryan SL, Lazar RM, Vry MS, et al. (2011) Prediction of motor recovery using initial impairment and fMRI 48 h poststroke. Cereb Cortex 21: 27122721. [Crossref]

38. Zhu LL, Lindenberg R, Alexander MP, Schlaug G (2010) Lesion load of the corticospinal tract predicts motor impairment in chronic stroke. Stroke 4: 910-915.

39. Teasell R, Bayona NA, Bitensky J (2005) Plasticity and reorganization of the brain post stroke. Top Stroke Rehabil 12: 11-26. [Crossref]

40. Behrens TEJ, Johansen-Berg H, Woolrich MW, Smith SM, Wheeler-Kingshott CA, et al. (2003) Non-invasive mapping of connections between human thalamus and cortex using diffusion imaging. Nature neuroscience 6: 750-757.

41. Cheng MY, Wang EH, Woodson WJ, Wang S, Sun G, et al. (2014) Optogenetic neuronal stimulation promotes functional recovery after stroke. Proceedings of the National Academy of Sciences 111: 12913-12918.

42. Fuglmeyer AR, Jääskö L, Leyman I, Olsson S, Steglind S (1975) The post-stroke hemiplegic patient. 1. a method for evaluation of physical performance. Scand $J$ Rehabil Med 7:13-31.

Copyright: (C2018 Ueda R. This is an open-access article distributed under the terms of the Creative Commons Attribution License, which permits unrestricted use, distribution, and reproduction in any medium, provided the original author and source are credited. 